Continents manuscrits

Génétique des textes littéraires - Afrique, Caraïbe,

diaspora

12 | 2019

Génétique et anthropologie

\title{
Le terrain comme fiction
}

Jean-Loup Amselle

\section{OpenEdition}

\section{Journals}

Édition électronique

URL : https://journals.openedition.org/coma/3587

DOI : $10.4000 /$ coma.3587

ISSN : 2275-1742

\section{Éditeur}

Institut des textes \& manuscrits modernes (ITEM)

\section{Référence électronique}

Jean-Loup Amselle, «Le terrain comme fiction », Continents manuscrits [En ligne], 12 | 2019, mis en ligne le 12 février 2019, consulté le 12 janvier 2023. URL : http://journals.openedition.org/coma/3587 ; DOI : https://doi.org/10.4000/coma.3587

Ce document a été généré automatiquement le 12 janvier 2023.

\section{(c) (i) ()}

Creative Commons - Attribution - Pas d'Utilisation Commerciale - Pas de Modification 4.0 International - CC BY-NC-ND 4.0

https://creativecommons.org/licenses/by-nc-nd/4.0/ 


\title{
Le terrain comme fiction
}

\author{
Jean-Loup Amselle
}

1 Quand Nicolas Martin-Granel m'a proposé d'intervenir dans ce séminaire, je me suis demandé ce que j'allais bien pouvoir raconter à des littéraires, peu au fait d'anthropologie, et surtout de l'histoire de l'anthropologie, et je me suis dit que «le terrain comme fiction » pouvait être un bon thème. Je m'appuierai en partie sur le paratexte.

2 Alors que les sciences sociales sont accusées par les pouvoirs de favoriser «l'excuse ", en mettant l'accent sur les causes sociales et économiques des comportements délictueux, le terrain et l'enquête de terrain sont revendiqués par les anthropologues, et les chercheurs en sciences sociales en général, comme l'alpha et l'omega de leurs disciplines, le moyen privilégié de contrer la doxa, l'idéologie dominante, en rendant compte des pratiques sociales effectivement observées.

\section{Le terrain scientifique}

3 Seligman (1873-1940), le maître de Malinowski, pratiquait l'ethnographie itinérante, se rendant d'île en île, dans le détroit de Torres entre la Nouvelle Guinée et l'Australie. Ce qui était approprié d'une certaine façon au phénomène du kula, ce système de don et de contre-don entre populations des différentes îles. Il faisait un peu comme Griaule dans un premier temps, lors de son expédition Dakar-Djibouti, avant de s'être sédentarisé à Sangha, en pays dogon, pour sa mission suivante. Malinowski a opté, en partie, à son corps défendant (assigné à résidence en tant que sujet autrichien pendant la guerre de 1914 en territoire du Commonwealth, Nouvelle Guinée australienne), pour l'étude « insulaire », intensive et localisée d'une communauté, celle des Trobriandais du détroit de Torres, par l'«observation participante». En gros, il s'agit d'une expérience de laboratoire (sélection d'un certain nombre de variables, méthode inspirée de Galton) menée en plein air par un anthropologue blanc sur des «indigènes", donc de l'imposition d'un savoir/pouvoir (parenté, mythes) sur des populations muettes. 


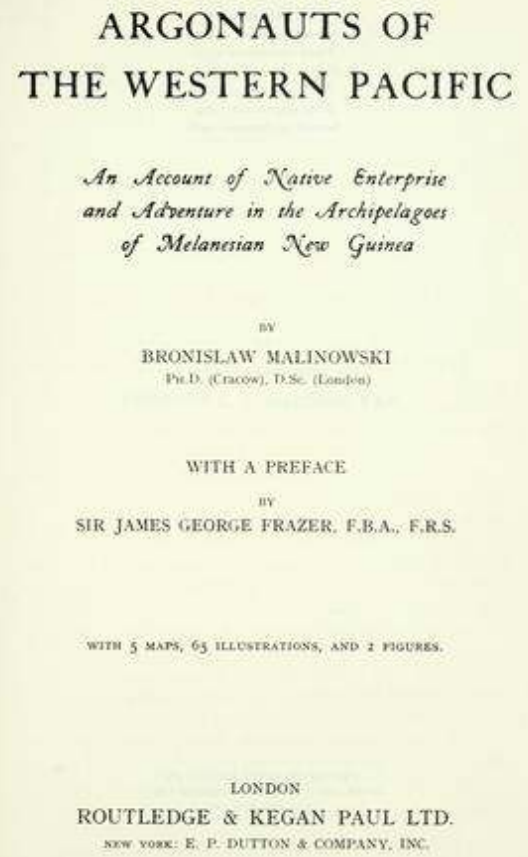

4 La procédure en avait été codifiée de façon précoce par les Notes and Queries on Anthropology ( $1^{\text {re }}$ édition en 1874 , suivie de nombreuses autres éditions). J'en ai lu un exemplaire édité dans les années 1960. Ce livre de conseils aux anthropologues débutants déroule toute une stratégie pour traquer, assujettir de façon optimale le « sauvage ». Où planter sa tente dans le village, à l'écart des différents clans et lignages envisagés comme des groupes de pression? 
NOTES AND QUERIES

\section{ANTHROPOLOGY}

rDitro XOR rII

COUNCIL OF THE ANTHROPOLOGICAL

INSTITUTE

sx

JOHN GEORGE GARSON, M.D.,

$$
\triangle X D
$$

CHARLES HERCULES READ, F.SA.

Second Edition.

LOXDox:

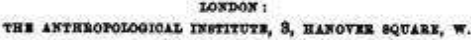
And at all Bookedtere.

1892.

PRICE FTVE SHILLINGA.

\section{L'indigène se rebiffe}

Qui est habilité à représenter l'Autre? Ou plutôt n'est-on habilité qu'à se représenter soi-même? Il existe désormais une critique de la scission du sujet et de l'objet, de l'objectivité anthropologique par les post-coloniaux et par les premiers concernés. Les " racisés » reprochent notamment aux anthropologues de les étudier comme dans un « zoo humain ». À cela est lié le problème de la restitution de l'information prélevée et traitée par l'anthropologue, restitution qui s'avère le plus souvent difficile, voire impossible en raison de visions discordantes entre l'anthropologue qui critique les sources et les militants qui s'en emparent en en faisant un marqueur politique...

Une des solutions à cette contradiction est: le passage de l'Autre côté (going native). L'anthropologue fait ainsi corps avec l'objet de sa recherche.

\section{Le terrain œdipien ou les affinités électives}

Autrefois, le terrain était choisi par un maître. Lévi-Strauss a ainsi envoyé Godelier en Nouvelle-Guinée parce que ce territoire abritait l'une des populations jugées parmi les plus «primitives » du globe. Il a fait de même avec Descola qui est parti enquêter chez les Jivaros d'Équateur. Balandier, pour sa part, envoyait un géographe ou un économiste et un anthropologue étudier une " communauté rurale »: Remy et Cartry chez les Gourmantché du Burkina Faso, Meillassoux et Deluz chez les Gouro de Côted'Ivoire, Copans et une équipe d'économistes et de géographes chez les Mourides ruraux du Sénégal, etc. Parfois, le choix du terrain était le fruit du hasard ou de contraintes institutionnelles. Ainsi Pierre Étienne devait travailler chez les Agni de 
Côte-d'Ivoire, mais l'Orstom avait besoin de lui pour enquêter chez les Baoulé dans le cadre d'une opération de développement et il est devenu ainsi un spécialiste des Baoulé, chez lesquels il a passé des années. C'était l'époque du «Dans mon village, ça se passe comme ça ». De toute façon, il faudrait faire une étude systématique sur le choix du terrain par les anthropologues.

Pour moi, ça ne s'est pas passé ainsi. J'ai découvert Meillassoux au milieu des années 1960 au séminaire de Balandier avec lequel je me suis inscrit en thèse. J'ai été séduit par ses idées et il m'a intégré au sein de son équipe de recherche sur les "Systèmes économiques africains précoloniaux»: esclavage, monopole souverain, commerce à longue distance, etc.

Dans le cadre de ce projet, nous étions amenés à dépouiller l'ensemble des relations de voyage traitant de l'Afrique précoloniale : auteurs arabes, Mungo Park, René Caillié, Binger, Heinrich Barth, et d'autres moins connus.

J'ai été frappé alors par l'importance des relations entre sociétés africaines à l'époque précoloniale et notamment par la force du commerce à longue distance en Afrique de l'Ouest (Hausa, Dioula, etc.), ce que je nommerai plus tard les «chaînes de sociétés ". J'ai donc choisi, en apparence pour des raisons purement scientifiques, d'étudier un groupe de commerçants à longue distance opérant depuis l'époque précoloniale et poursuivant ses activités jusqu'à l'époque contemporaine (esclaves, chevaux puis cola, bétail, etc.) à travers des réseaux marchands internationaux (Côte-d'Ivoire, Sénégal, Mali, Burkina Faso). C'est ce qui a fait l'objet de mes premières enquêtes de terrain et a donné lieu à ma thèse de $3^{\mathrm{e}}$ cycle, comme on disait alors, publiée ensuite sous le titre Les Négociants de la Savane (1977).

Fig. 3 : Les Négociants de la savane

\section{jean-loup amselle}

\section{LES NEGOCIANTS}

\section{DE I.A SAVANE}

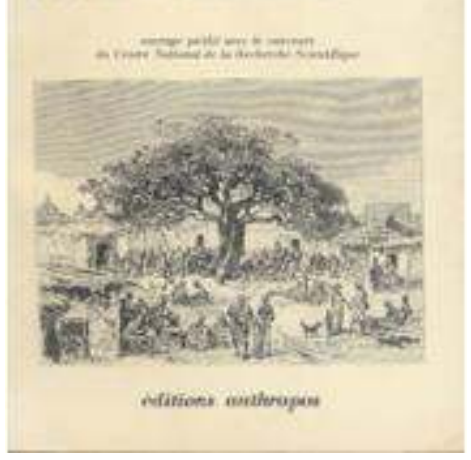

11 En réalité, dans cette thèse et dans ce livre, je réglais mes comptes avec mon père qui était commerçant de chaussures et qui avait tenté d'interrompre mes études alors que j'étais au lycée pour me contraindre à travailler avec lui. Mes amis modifièrent ainsi le titre de ce livre en "Les Négociants de la savate». À travers cette étude d'une "diaspora marchande ", selon l'expression de l'anthropologue Abner Cohen à propos des commerçants de cola hausa du Nigeria, c'est aussi à mon statut de juif que je m'adressais. Et l'on pourra retrouver dans mon livre des références à l'Israël que 
représente le village de Madina pour les Diawara, village dont est originaire la chanteuse malienne mondialement connue Fatoumata Diawara. Autre référence, le statut de ces marchands "forgerons ", c'est-à-dire " castés ", faisait écho à mon propre statut de juif français certes assimilé mais néanmoins en porte-à-faux par rapport à la société française, de "métèque » au sens grec (d'étranger résident) ou "métis » en quelque sorte si l'on veut bien voir dans ce concept éminemment flou, non pas un état mais une relation. On est en effet toujours le métis de quelqu'un.

Enfin l'histoire même de ce groupe constitué de gens qui, grâce à l'utilisation de la parenté à plaisanterie Peul-forgerons, s'étaient fait passer pour des forgerons pour échapper à l'esclavage et à la mort, renvoyait sans doute pour moi à ma propre condition d'individu contraint de dissimuler ses origines de "non Français de souche " à part entière. Amselle, ça ne fait pas français « de souche »!

Bref, l'identité ou plutôt les troubles de l'identité, pour paraphraser Judith Butler, je suis tombé dedans à la naissance et ce n'est sans doute pas un hasard si je me suis interrogé sur les changements d'identité et leur caractère éminemment métis ${ }^{1}$.

Toute ma carrière d'anthropologue, et même le choix de cette carrière, ce qu'on pourrait nommer l'« avant-terrain », comme vous parlez des " avant-textes », pourrait s'analyser de la sorte comme le produit d'une trajectoire purement personnelle.

15 J'ai commencé en effet par faire des études de droit et d'anglais pour faire plaisir à mon père, puis j'ai abandonné ces études pour me diriger vers la sociologie et l'ethnologie. Depuis, je n'ai pas quitté ce domaine mais j'y ai toujours œuvré de façon iconoclaste, à la fois par rapport à la notion de culture, à celle de langue et à celle d'Afrique.

16 Je me suis toujours senti mal à l'aise par rapport à la notion de culture telle qu'elle a été véhiculée, manipulée par l'anthropologie culturelle américaine, même si Ralph Linton, un disciple de Franz Boas, a lui-même apporté un bémol à cette vision culturaliste. Dans un article humoristique, il faisait ainsi le récit de la matinée d'un Américain en notant qu'après son petit déjeuner, celui-ci prenait son parapluie inventé en Inde, puis une fois installé dans son train, invention britannique, s'apprêtait à fumer une cigarette, inventée au Mexique, ou un cigare, inventé au Brésil, en lisant son journal, imprimé selon un procédé inventé en Allemagne sur du papier inventé en Chine. Après avoir parcouru un énième éditorial mettant en garde contre les terribles menaces que faisaient peser sur les institutions l'adoption des idées étrangères, il remerciait dans une langue indo-européenne une divinité hébraïque d'être $100 \%$ (système décimal inventé dans l'Antiquité par les Grecs) américain (dont le nom l'Amérique vient d'Amerigo Vespucci, géographe italien) ${ }^{2}$ !

17 Mais précisément, cet article, au-delà de son aspect généreux et ouvert, reproduit d'une certaine façon ce que je dénonce comme étant l'aporie du métissage, à savoir l'idée que pour métisser, il faut poser au départ l'hypothèse de cultures ou de langues discrètes (au sens mathématique du terme) et que l'on est donc renvoyé au point de départ.

18 Au fond, ma gêne à l'égard de la notion de culture provient du fait que, d'une part, elle renvoie les acteurs sociaux à leur origine supposée (comme on dit maintenant de quelqu'un qu'il a "des origines») et, d'autre part, parce qu'elle pose question à quelqu'un comme moi qui suis Français sans l'être tout à fait, ou qui en tout cas ne se sent pas tel, mais plutôt, comme je l'ai déjà dit, comme «métis». Il n'est donc pas douteux que j'ai projeté mes propres préoccupations, obsessions et fantasmes sur mon ou mes terrains maliens et que la déconstruction que j'ai entreprise des catégories 
ethniques ou autres provient de ma propre incertitude par rapport à ma propre société et à ma propre identité. Et ici, je me trouve en bonne compagnie avec Margaret Mead qui disait à peu près la chose suivante : quand on a des problèmes avec soi-même, on devient psychologue, quand on a des problèmes avec sa société, on devient sociologue et quand on a des problèmes avec soi-même et avec sa société on devient anthropologue...

Fig. 4 : Margaret Mead, Mœurs et Sexualité en Océanie, Paris, Plon, 1955

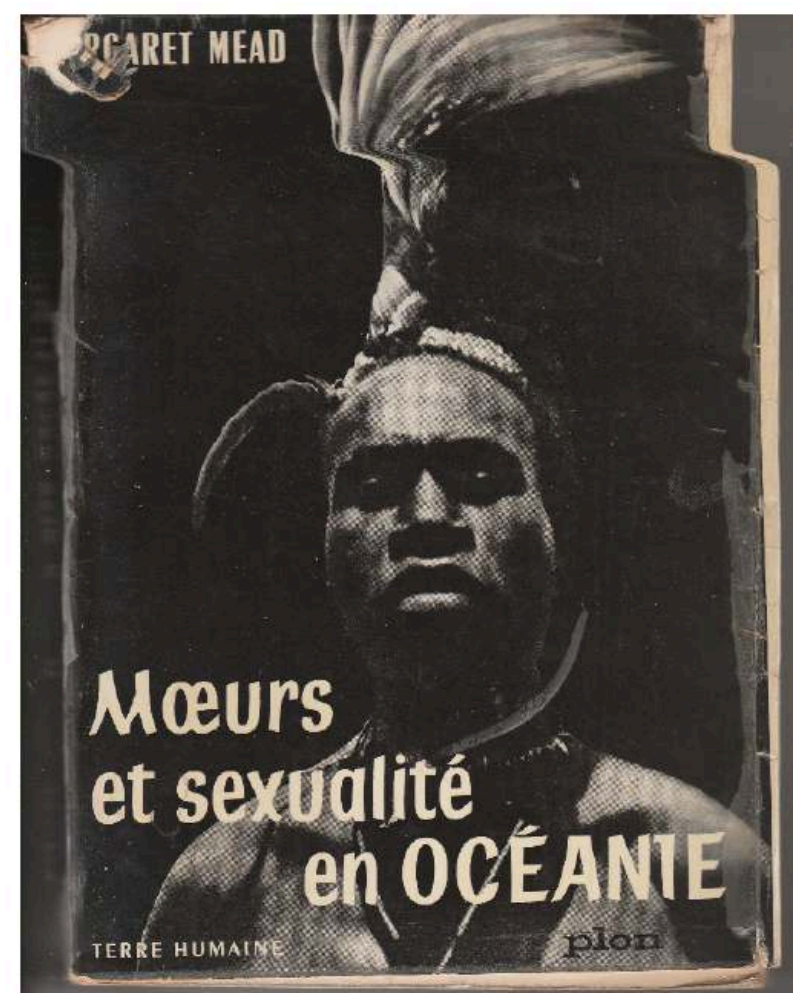

\section{Le terrain emplumé}

Cette référence à $M$. Mead me permet de passer à un autre aspect du thème du terrain comme fiction : ce que j'appellerai le terrain emplumé. Les idées de Margaret Mead sur la sexualité en Océanie ont été abondamment critiquées par l'anthropologue australien Derek Freeman ; il est en effet probable que Margaret Mead a largement fantasmé sur la liberté sexuelle dont auraient joui les jeunes Samoans et elle a sans doute projeté ses désirs de libération sexuelle sur cette population exotique. Ses livres ont connu un énorme succès parce qu'ils ont été patronnés par son maître Franz Boas et sa maîtresse (dans les deux sens du terme) Ruth Benedict. Mais c'est aussi parce qu'ils correspondaient à un horizon d'attente primitiviste d'une population américaine engoncée dans le puritanisme. Pour paraphraser Gide, je dirais donc que c'est avec de la mauvaise anthropologie qu'on fait de la bonne littérature, nonobstant le fait que Margaret Mead était dotée d'une bonne plume. Mais au-delà du style, qui fait beaucoup pour le succès de certains livres d'anthropologie - je pense en particulier ici à LéviStrauss ou à P. Clastres - ce qui est sans doute au cœur du succès des «block busters " anthropologiques, c'est une mise en scène primitiviste de certaines populations. Je dis d'ailleurs ça sans aucune animosité car je n'ai pas trop à me plaindre mais il est certain 
que la déconstruction de l'ethnie, les logiques métisses en tant que catégories contreintuitives ne sont pas vraiment porteuses, en tout cas moins porteuses que les mises en scène des populations censées être les plus "primitives" du globe, telles les Nambikwara du Brésil (Lévi-Strauss), les Baruya de Nouvelle-Guinée (Godelier), ou les Jivaro d'Équateur (Descola).

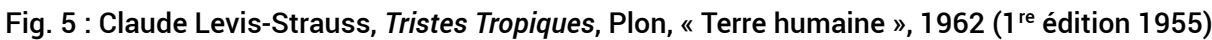

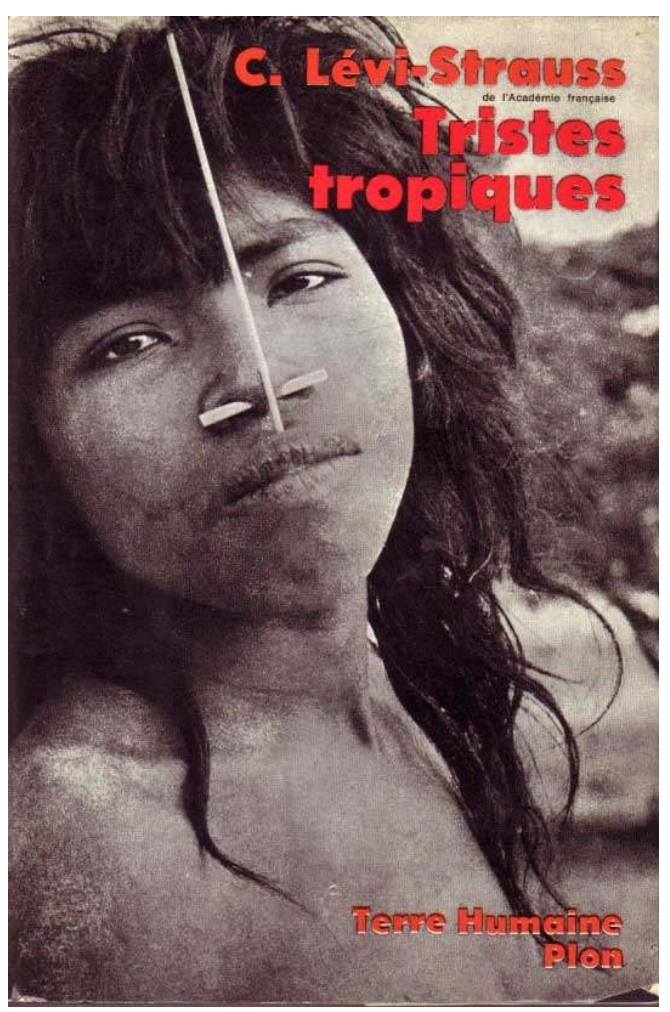

Fig. 6 : Maurice Godelier, La Production des grands hommes, Flammarion, « Essais », 1982

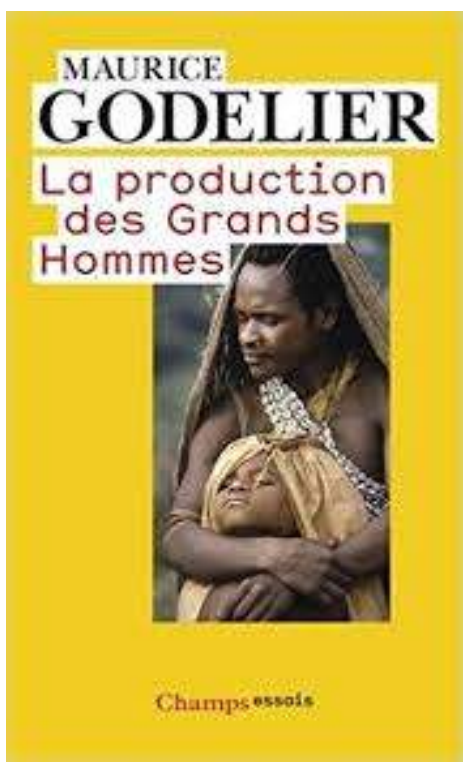


Fig. 7 : Philippe Descola, Les Lances du crépuscule, « Terre humaine », Plon, 1993

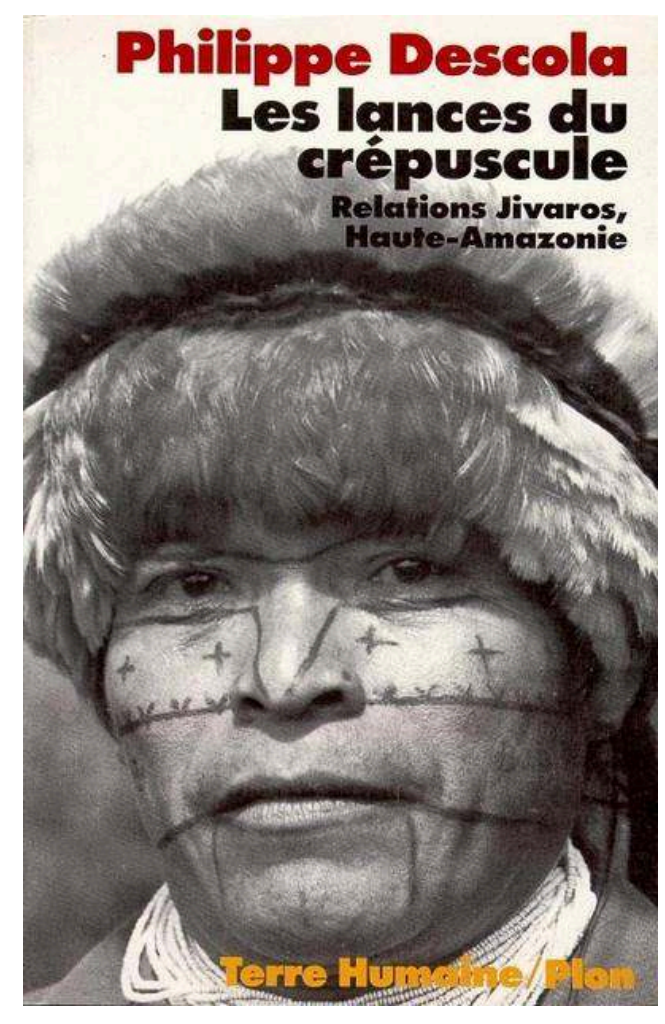

À cet égard, on comprend mieux la défaite institutionnelle du courant balandiériste par rapport au courant lévi-straussien, défaite due au déficit primitiviste du premier courant. Comment Balandier pouvait-il s'aligner avec ses Fang et ses Bakongo face aux Nambikwara et aux Caduvéo de Lévi-Strauss? Et s'il est certain que Tristes Tropiques (1955) est une œuvre littéraire de meilleure qualité qu'Afrique ambiguë (on dit même qu'Alfred Métraux qui avait été commandité par Jean Malaurie et "Terre humaine » pour écrire un livre du même type se serait suicidé pour n'avoir pu y parvenir), le succès phénoménal de Tristes tropiques doit sans doute en partie au rousseauisme, pour aller vite, d'ailleurs revendiqué par son auteur. 


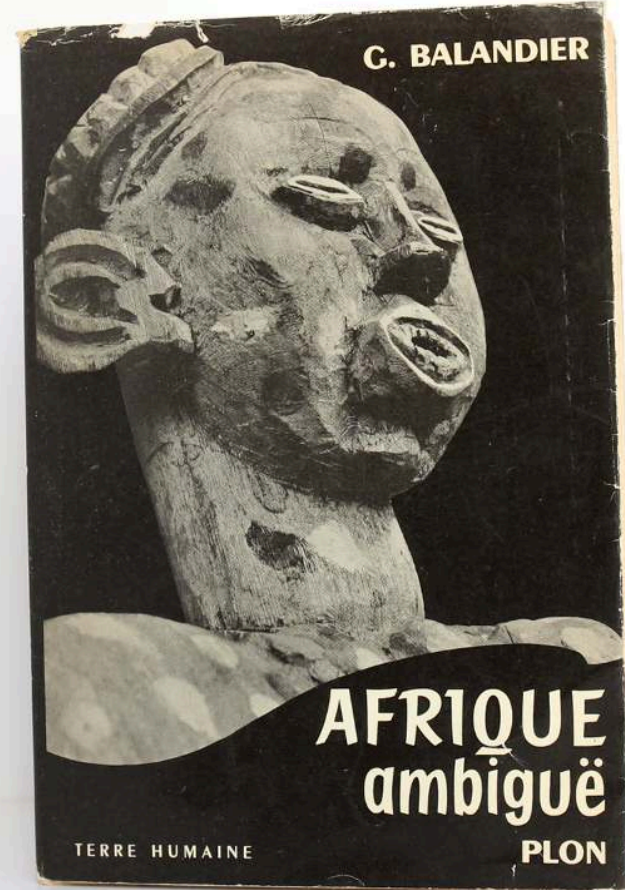

21 À cet égard, il faudrait faire un sort particulier à la collection "Terre humaine ", déjà évoquée, collection-phare du primitivisme anthropologique, dirigée pendant de longues années par Jean Malaurie et désormais par Jean-Christophe Ruffin, médecin, diplomate, écrivain et contempteur de la globalisation ${ }^{3}$. Je signale d'ailleurs à ce propos que l'un des mes amis, anthropologue amazoniste, s'est vu refuser un manuscrit par J. Malaurie parce qu'il était jugé trop contemporanéiste, c'est-à-dire pas assez primitiviste.

22 Tous ces thèmes, nous les avions abordés dans un ouvrage qui nous a valu pas mal d'ennemis : Le Sauvage à la mode (1979), où étaient critiqués Lévi-Strauss, Clastres, Sahlins, Jaulin mais aussi Turnbull (mais on aurait dû aussi traiter de Castaneda) et les idées libertaires de "société contre l'État», celles d'«ethnocide», de "première société d'abondance ", d'idéalisation des sociétés de chasseurs collecteurs. Mais le sauvage n'était pas envisagé seulement sous l'angle de sa "bonté » supposée; sa "méchanceté " était également pointée, chez les Iks décrits par Turnbull et réinterprétés dans le cadre d'un véritable « théâtre de la cruauté » par Peter Brook aux Bouffes du Nord. C'est le «dark side » de l'anthropologie africaniste qui fait écho à des œuvres littéraires comme Au cœur des ténèbres de Conrad, Voyage au bout de la nuit de Céline ou Le coup de lune de Simenon ou encore Le Devoir de violence de Yambo Ouologuem. Mais cela renvoie aussi à toute la littérature produite autour du génocide rwandais et qui véhicule aussi, d'une certaine façon, l'idée d'une sauvagerie africaine. 
Fig. 9 : Jean-Loup Amselle, Le Sauvage à la mode, Le sycomore, 1979

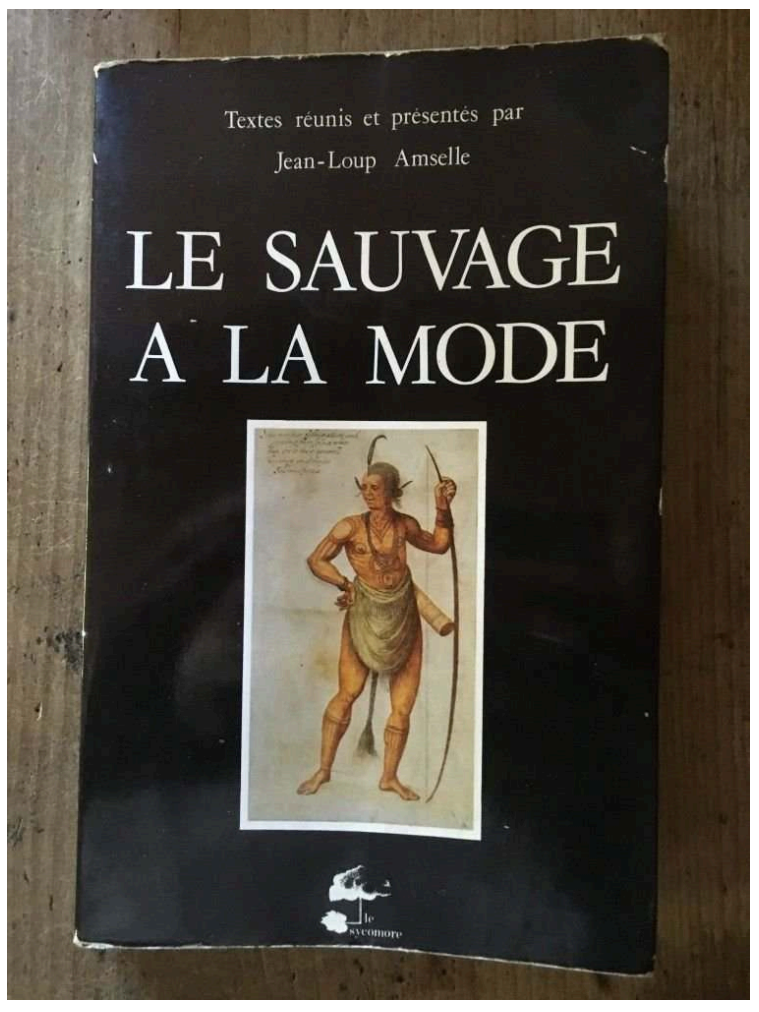

23 Je fais une référence à Artaud car, là aussi, il faudrait reprendre de façon critique ses écrits sur les Tarahumaras du Mexique (chez lesquels il ne s'est d'ailleurs peut-être jamais rendu), écrits d'ailleurs à l'origine de certaines vocations anthropologiques, tout comme il faudrait reprendre les écrits de Victor Segalen qui ont fortement inspiré Édouard Glissant.

Fig. 10 : Antonin Artaud, Les Tarahumaras, Folio, 1987 ( $1^{\text {re }}$ édition en 1945)

\section{Antonin Artaud \\ Les \\ Tarahumaras}

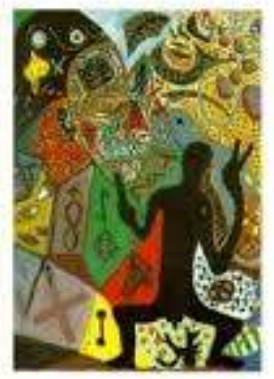

$$
\text { 6o. [] main }
$$

Bref, il existe une circulation réciproque entre littérature et anthropologie parce que l'anthropologie, la monographie anthropologique est largement la mise en récit d'une 
population donnée et pas seulement la description objective d'une communauté. James Clifford, de ce point de vue, a bien montré les liens existant entre Joseph Conrad et Bronislaw Malinowski et il cite à ce propos une phrase du Journal d'ethnographe qui fait écho à Au cœur des ténèbres : «Par moment, j'étais furieux contre eux, particulièrement parce que, lorsque je leur eus donné une portion de tabac, ils s'en étaient tous allés. En gros mes sentiments à l'égard des indigènes allaient dans le sens de "Exterminez les brutes"” ». Dans ce Journal, Malinowski qualifie d'ailleurs les Trobriandais de «nègres » (niggers).

Fig. 11 : Bronislaw Malinowski, Journal d'ethnographe, Le Seuil, 1985 (1 ${ }^{\text {re }}$ édition en 1967)

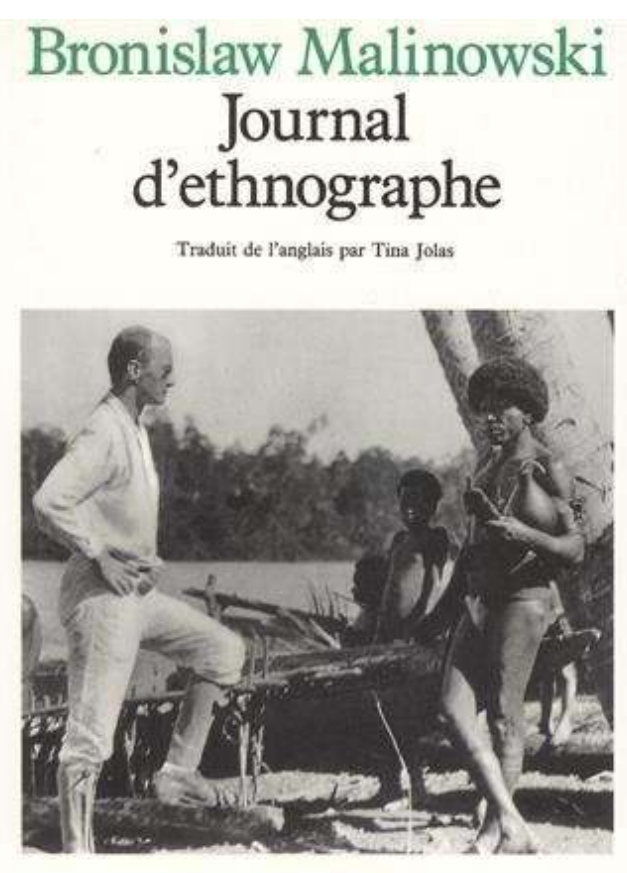

Recherches anthropologiques/Seuil 
Fig. 12 : Joseph Conrad, Au cœur des ténèbres, Folio bilingue, 1996 ( $1^{\text {re }}$ édition 1899)

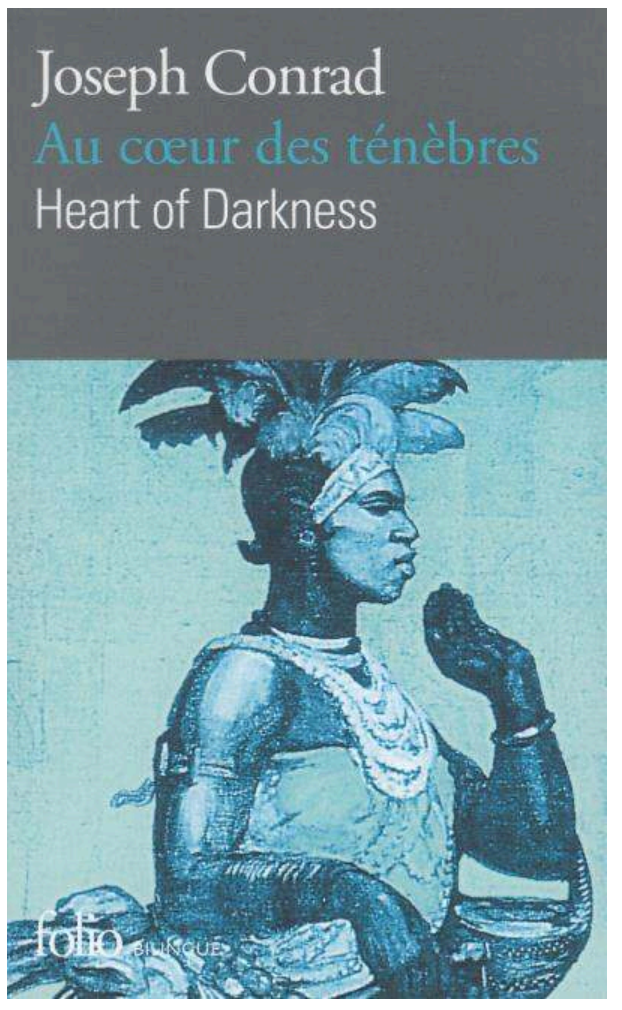

Il n'existe donc pas de hiatus entre littérature et anthropologie puisque l'anthropologie est non seulement une science humaine mais également une discipline littéraire au sens où la monographie est un récit relatant une expérience vécue et transcrite-écrite par un auteur, l'anthropologue. La question est donc la suivante: le Journal d'ethnographe de Malinowski, écrit en polonais et non destiné à être publié, est-il plus vrai, plus fidèle à son expérience chez les Trobriandais que Les Argonautes du Pacifique occidental écrit en anglais, ouvrage scientifique destiné à asseoir sa carrière institutionnelle? Les notes de terrain doivent-elles être considérées comme des « avant-textes » analogues aux premières ébauches d'un roman?

Pour ma part, je refuse que mes notes de terrain fassent l'objet d'une sorte de "génétique anthropologique ». Je réclame le droit au non bénéfice d'inventaire. Selon moi, l'expérience anthropologique est unique, elle n'est pas reproductible et je réclame donc le droit à l'« invention » du terrain, au sens d'y avoir découvert un trésor, le droit de n'être jugé que sur pièces, une fois le travail fini et livré dans sa forme définitive au public. Bref, je réclame le droit à la prescription.

\section{Conclusion : la scène primitive}

L'autre ou l'Autre, c'est quoi? C'est l'autre exotique ou l'exotisme de soi? Que va chercher l'anthropologue aux antipodes, la sauvagerie des autres ou sa propre sauvagerie, celle de l'autre scène ? Comment être sûr que lorsque je parle des autres, je ne parle pas de moi-même? Et lorsque l'anthropologue met en scène les primitifs, n'est-ce pas de l'autre scène qu'il parle, de sa propre anthroposcène, avec un « $\mathrm{s}$ "? Vieux problème, abordé par Freud dans Totem et tabou, ce roman des origines qui est sans doute le roman de sa propre origine fantasmée. 
toute la tradition philosophique et littéraire européenne suppose, postule que les cultures exotiques sont là pour donner une leçon de civilisation à l'Europe, puisque ces sociétés, africaines en l'occurrence, sont, comme le dit Césaire, «civilisées » jusqu'au bout des ongles.

est ainsi devenu gardien de la nature, écologiste, et à la limite ennemi de cette même nature depuis son entrée en scène sur la terre, bref depuis l'anthropocène, sans « $\mathrm{s}$ » cette fois, mère de l'effondrement à venir. La racine de tous les maux ne gît plus dans le capitalisme, dans l'exploitation éhontée des hommes et de l'environnement par les multinationales minières mais dans le viol de la Terre-Mère, de la Pachamama ou de Gaïa, conception qui donne à la Terre le statut d'être vivant.

Il ne s'agit donc plus de rendre la "Terre plus humaine " pour faire référence à la collection précitée, mais au contraire de la déshumaniser, de faire en sorte que l'homme ne soit plus maître et possesseur de la nature (Descartes), mais au contraire son serviteur, voire qu'il disparaisse. C'est d'ailleurs ce qui transparaît dans l'œuvre de Lévi-Strauss, le souhait de disposer d'un monde d'avant l'homme là où l'on n'entendrait que le bruit des bêtes sauvages et le chant des oiseaux...

\section{NOTES}

1. Cf. Jean-Loup Amselle, Logiques métisses. Anthropologie de l'identité en Afrique et ailleurs, Paris, Payot, 1990. Et je pourrais dire, en un sens, pour paraphraser Flaubert, que « Logiques métisses », c'est moi !

2. R. Linton, « One hundred per cent American », American Mercury, $n^{\circ}$ 40, avril 1937, p. 427-429.

3. Cf. David Couvidat, «Résister face à la mondialisation », Revue critique de fixxion française, 2017.

4. Clifford James, «De l'ethnographie comme fiction. Conrad et Malinowski», Études rurales, $\mathrm{n}^{\circ}$ 97-98, 1985, p. 47-67.

\section{RÉSUMÉS}

$\mathrm{Au}$ cours de cet exposé, axé sur l'histoire de l'anthropologie et l'expérience personnelle de l'intervenant, seront abordés successivement les thèmes suivants: le terrain scientifique, l'indigène se rebiffe, le terrain œdipien, le terrain emplumé, la scène primitive.

$\operatorname{xxxxxxxx}$ 
INDEX

Mots-clés : Anthropologie, Afrique, Malinowski, Lévi-Strauss, Balandier, Meillassoux

Index géographique : Afrique

\section{AUTEUR}

\section{JEAN-LOUP AMSELLE}

Jean-Loup Amselle est anthropologue et directeur d'études à l'EHESS. Il a effectué ses travaux de terrain au Mali, en Côte-d'Ivoire et en Guinée, sur les Peuls, les Bambaras et les Malinkés. Il en a dégagé une approche critique et déconstructiviste dans les domaines de l'identité, de l'ethnicité et du métissage allant à l'encontre du primitivisme anthropologique et des idées post- et décoloniales. Il a publié, entre autres, Le Sauvage à la mode (Le Sycomore, 1979), Au cour de l'ethnie (La Découverte, 2005, 1re éd. 1985, avec avec E. M’Bokolo), Logiques métisses (Payot, 2010, $1^{\text {re }}$ éd.

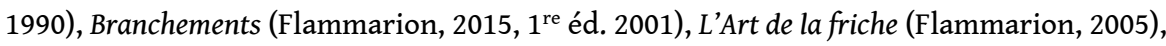

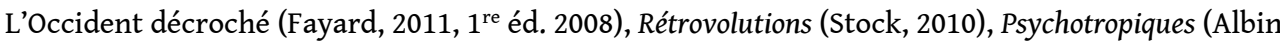
Michel, 2013), Islams africains (Le Bord de l'eau, 2017, avec S. B. Diagne), En quête d'Afrique(s) (Albin Michel, 2018). 